\title{
Phobos and Deimos CCD observations ${ }^{\star}, \star \star$
}

\author{
C. H. Veiga \\ Observatório Nacional, Rua General José Cristino 77, Bairro Imperial de São Cristóvão Cep:20921-400 Rio de Janeiro, Brazil \\ Coordenação de Astronomia e Astrofísica \\ e-mail: cave@on.br
}

Received 1 February 2008 / Accepted 14 May 2008

\begin{abstract}
Aims. This paper give positions of Martian satellites system from CCD observations obtained at the Cassegrain focus of a $1.6 \mathrm{~m}$ reflector for the years 1995 and 2003.

Methods. These positions were reduced using the scale and orientation parameters, determined from star field positions of Uranus and Pluto systems' observations that were made in the same night as Mars observations.

Results. They are compared with the theoretically calculated positions from JPL Development planetary Ephemeris - DE405. The standard deviations of the residuals, observed minus calculated positions, right ascension and declination of Phobos and Deimos, respectively are $\sigma=0$.' $^{\prime} 079$ and $\sigma=0$.' $^{\prime} 072$.
\end{abstract}

Key words. celestial mechanics - astrometry - ephemerides

\section{Introduction}

The modern observations of the main planets and their satellites are accomplished by spacecraft missions. These observations are important because the spacecraft flybys allow us to see the planet's physical characteristics in greater detail. From the Voyagers spacecraft missions in the 70's until the New Horizons spacecraft mission, a new view of the Solar System has been presented. For example, around Jupiter 63 satellites are now known (Veiga 2006); the important details of the Mars surface have been revelated; and the first asteroidal satellite Dactyl was discovered during the 1993 flyby of 243 Ida of the Galileo spacecraft (Chapman et al. 1995).

Recently, thirty-six accurate astrometric positions of Phobos and Deimos, using the onboard camera Super Resolution Channel on Mars Express spacecraft (launched on 2 June 2003), were published (Oberst et al. 2006). The accuracy of these observations greatly exceeds the terrestrial data. However, the Earthbased observations have the advantage of longer observation orbit arcs, leading perhaps to a greater orbital precision. Moreover, the spacecraft missions need high accuracy ephemeris for their preparation to perform close approches to planetary bodies.

Therefore, the spacecraft and Earth-based observations complement each other nicely in the exploration of the Solar System bodies.

The determination of Mars' positions satellites Phobos and Deimos accuracy, from Earth-based observations has always been very difficult, due to the several problems their observations present. These satellites are bodies with visual magnitudes

* Based on observations made at the Laboratório Nacional de Astrofísica/MCT-Itajubá-Brazil.

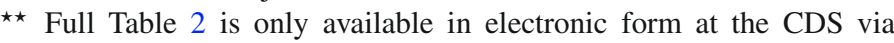
anonymous ftp to cdsarc.u-strasbg.fr (130.79.128.5) or via http://cdsweb.u-strasbg.fr/cgi-bin/qcat?J/A+A/487/755 of 11.8 and 12.9, respectively, while the magnitude of the planet is -1.5 , for two years observations, 1995 and 2003. Due to the great difference in magnitude between them, and their orbits to be very close to the planet, 8.178 and $17.750 \mathrm{~km}$, corresponding to the mean distance for 1995 and 2003 missions, the strong diffused light from the planet causes the destruction of the maximum density that points out the center of satellite's images. It can also be affected by systematic error generated by the addition of a non-horizontal background, and give a position shift toward the planet's center (Veiga \& Martins 1995). Moreover, the planet may move during the image acquisition, thus several images are elongated.

In order to minimize these effects, was used the following procedure for all observations of Mars' satellite system:

(i) a diaphragm with eight circular apertures between the secondary support vanes, because the diffraction spikes on Mars' image can occult the images of Phobos and Deimos;

(ii) a coronagraph with the astrometric purpose of imaging satellites near planets; and

(iii) one two-dimensional elliptic Gaussian to determine the images' centers to improve the accuracy of the measured satellites' position.

\section{The observations and satellite image center measurements}

All 185 observations, accomplished in 1995 and 2003, were carried out at the classical Cassegrain long focus of the $1.6 \mathrm{~m}$ Ritchey-Chretien reflector at the Laboratório Nacional de Astrofísica (LNA/MCT) Itajubá-Brazil, with focal length of $15.8 \mathrm{~m}$, and scale in focal plane of $13^{\prime \prime} / \mathrm{mm}$. The geographical coordinates are $\lambda=3^{\mathrm{h}} 02^{\mathrm{m}} 19^{\mathrm{s}}$ west, $\phi=22^{\circ} 32^{\prime} 04^{\prime \prime}$ south and $h=1872 \mathrm{~m}$. 
Table 1. Date and number of CCD observations and observed hours per night. The two last columns are the mean distance (in arcseconds) of the satellites from Mars.

\begin{tabular}{ccccc}
\hline \hline Nights & Number of & Number of & Distance from Mars \\
& CCD observations & observed hours & Phobos & Deimos \\
\hline $09 / 06 / 1995$ & 24 & $1^{\mathrm{h}} 52^{\mathrm{m}}$ & & $21^{\prime \prime} .0$ \\
$12 / 06 / 1995$ & 10 & $1^{\mathrm{h}} 44^{\mathrm{m}}$ & & $18^{\prime \prime} 8$ \\
$21 / 08 / 2003$ & 64 & $1^{\mathrm{h}} 33^{\mathrm{m}}$ & $31^{\prime \prime} 7$ & $74^{\prime \prime} 7$ \\
$23 / 08 / 2003$ & 87 & $2^{\mathrm{h}} 13^{\mathrm{m}}$ & 29.6 & $76^{\prime \prime} 7$ \\
\hline Total & 185 & $7^{\mathrm{h}} 22^{\mathrm{m}}$ & $30 .{ }^{\prime} 5$ & $65^{\prime \prime} .8$ \\
\hline
\end{tabular}

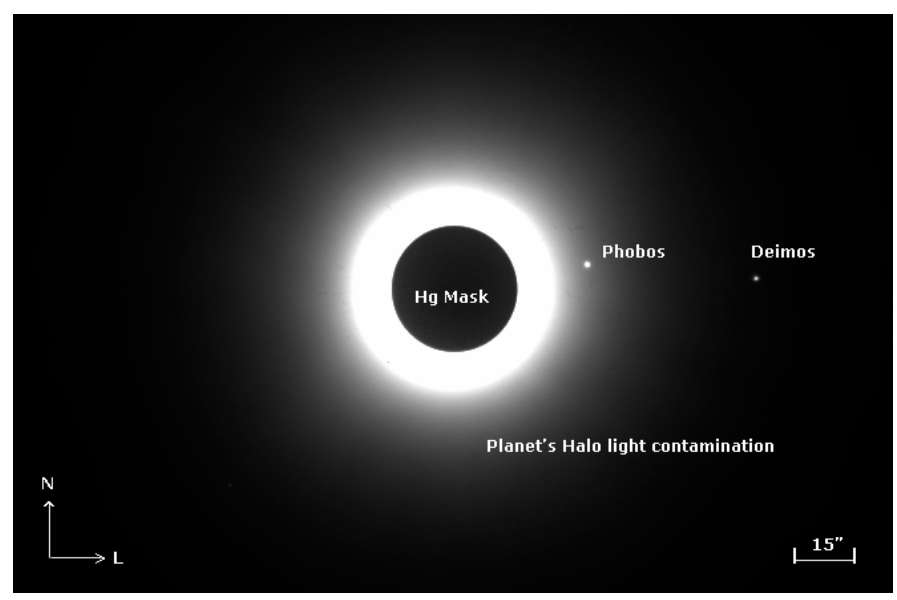

Fig. 1. CCD frame observed at 23/08/2003, $4^{\mathrm{h}} 7^{\mathrm{m}} 27^{\mathrm{s}}$ UTC. Exposure time $1^{\mathrm{s}}$. CCD dimension $1050 \times 1050$ square pixels.

In Table 1, considering the orbital periods of Phobos $=0.32$ and Deimos $=1.26$ days (Lynch et al. 2007), for all nights the mean number of observed hours cover $24 \%$ and $6 \%$ Phobos and Deimos orbits, respectively.

In this work two CCD types to take the 185 images: in 1995 , was used an array of $770 \times 1152$ square pixels corresponding to $22.5 \mu \mathrm{m}, 0.294$ on the sky. In 2003, an array was utilized of $1050 \times 1050$ square pixels corresponding to $24 \mu \mathrm{m}, 0.312$ on the sky. No filter and no anti-blooming CCD system in these observations were used, and the exposure time was equal to $1 \mathrm{~s}$.

We can see, in Fig. 1, that the observation does not contain the four diffraction spikes present in previous images due to the support of the secondary mirror. An external eight hole mask was placed on the entrance pupil (Veillet \& Ratier 1980). The decrease of nearly $40 \%$ in the useful area of the primary mirror was compensated by an increase in the exposure time.

In the 1995 observations, the images of the planet were hidden by a round mask put on the CCD window near the CCD surface. In the 2003 observations, was used a new coronagraph especially designed to the astrometric purpose of imaging satellites near planets. A classical Lyot mask (Lyot 1939) was substituted by an electronic system with a variable-diameter occulting spherical disk (Hg mask) (Bourget et al. 2001). The advantage of the new coronagraph system over the fixed mask is that, depending on the local meteorological conditions (seeing), we can vary the diameter of $\mathrm{Hg}$ mask. This allows us to hide the image of the planet with more efficiency. In spite these precautions, we can see in Fig. 1 that the sky around the planet remained very contaminated due to the strong light diffusion from Mars images.

To improve the accuracy of the positions measured for the satellites, one two-dimensional elliptic Gaussian, which takes into account the asymmetry of the images, was used for the determination of their centers. To fit the Gaussian parameters a non-linear least-square procedure was used. Starting from Bevington (1969), an algorithm for two dimensions was developed. The two-dimensional elliptic Gaussian, added to constant sky background, is given by:

$\boldsymbol{D}(x, y)=C^{\prime}+h \mathrm{e}^{-\frac{1}{2}\left[A\left(x-x_{0}\right)^{2}+B\left(x-x_{0}\right)\left(y-y_{0}\right)+C\left(y-y_{0}\right)^{2}\right]}$

where the initial values of the seven unknown parameters are: $C^{\prime}$, the value of the sky background, which is the mean value of the counts in the matrix corners; $h$, the Gaussian height value of the counts in the matrix; $\left(x_{0}, y_{0}\right)$, the coordinates of the Gaussian function center, corresponding the marginal distributions; and $A, B, C$, the constants associated to the variance of the Gaussian, which are the inverses of the half-width at the half-height in some directions of the satellites' images. Finally, $(x, y)$ are the pixel coordinates.

The determination of the center is considered accurate when the difference between the residuals of the fit is smaller than $1 \%$ of the last iteration residual values, and when the difference between the residuals of the center determination is smaller than 0.2 pixels. The trimming process (Chiu 1977) was also used to eliminate the pixels from an elliptic area at a larger distance than 2.5 times the semi-major axis of the ellipse. The errors of the centering procedure was 0.010 for the two satellites.

\section{Astrometric calibration}

Due to the background light gradient on the sky, it was not possible to use the position of star field in the Mars for the classical astrometric calibration. All observations had only limited numbers of faint stars in the field with magnitudes between 18 and 20 .

In order to solve this problem, the stars' positions of the observations of Uranus and Pluto were used in 1995 and 2003, respectively. The observations of Mars, Uranus and Pluto systems were accomplished alternately on the same night. The CCD orientation was maintained. The origin of the coordinate system was located in Mars. Colas et al. (1992) used the same process, but such fields were those of M15 globular cluster. In the present paper, for each night of observation in 1995 several CCD images of Mars and Uranus were observed alternately. The same procedure was used for Mars and Pluto observations in 2003.

For the Uranus and Pluto star field, the classical process of astrometric calibration was used (Veiga 2006). Utilizing the stars positions (measured and from catalog) of the field of each CCD frame observation of Uranus or Pluto, the least-squares procedure was used to determine the scale and orientation parameters. The procedure was applied to the mean parameters of each night for the determination of the equatorial coordinates of Phobos and Deimos.

In the list, available only in electronic form at the CDS, the topocentric observed positions of Phobos and Deimos are referred to a mean equator and equinox J2000 system. The mean instant of observation is given in Julian Date (UTC). The coordinates right ascension and declination are given in degree and fraction.

Starting from the left column of the list, we have: the name of the satellites, the julian date of the observation, the topocentric observed right ascension, and the declination. Table 2 provides a brief excerpt of the electronic list.

\section{Analysis of Phobos and Deimos observations}

In this section, the observed positions of Phobos and Deimos satellites are compared with their theoretical positions 
Table 2. Excerpt from the observations list, available only in electronic form at the CDS. From left to right: the name of satellites; the time in Julian Date (UTC); and the right ascension and declination coordinates, given in degree and fraction. These topocentric observed positions of two satellites refer to a mean equator and equinox J2000 system.

\begin{tabular}{cccc}
\hline \hline Satellite & Date & Right ascension & Declination \\
\hline Phobos & 2452872.752084491 & 341.2239091 & -15.1838285 \\
Deimos & 2452872.752084491 & 341.1953815 & -15.1843957 \\
Phobos & 2452872.754208333 & 341.2234622 & -15.1838686 \\
Deimos & 2452872.754208333 & 341.1946309 & -15.1846936 \\
Phobos & 2452872.755233796 & 341.2232502 & -15.1838743 \\
Deimos & 2452872.755233796 & 341.1943158 & -15.1848254 \\
\hline
\end{tabular}

Table 3. Observed minus calculated statistics for four major Uranus satellites in 1995 and Pluto observations in 2003. For Uranus satellites reductions, we have ${\overline{(\mathrm{O}-\mathrm{C})_{x}}}_{x}, \overline{(\mathrm{O}-\mathrm{C})_{y}}$ and $\left(\sigma_{x}\right),\left(\sigma_{y}\right)$ that are the means and the standard deviations for the residuals referred to Oberon satellite. The results ${\overline{(\mathrm{O}-\mathrm{C})_{\alpha}}}_{,}, \overline{\mathrm{O}-\mathrm{C})_{\delta}}$ and $\left(\sigma_{\alpha}\right),\left(\sigma_{\delta}\right)$ are for Pluto residuals topocentric coordinates (the units are arcseconds). $\bar{N}_{\text {obs }}$ is the mean number of observations, and $\bar{N}_{\text {stars }}$ the mean number of reference stars used in the astrometric calibration of Uranus and Pluto's system.

\begin{tabular}{|c|c|c|c|c|}
\hline & $\begin{array}{c}\text { Four major } \\
\text { Uranus' satellites }\end{array}$ & & & \\
\hline $\begin{array}{c}\overline{(\mathrm{O}-\mathrm{C})_{x}} \\
\left(\sigma_{x}\right)\end{array}$ & $\begin{array}{c}{\overline{(\mathrm{O}-\mathrm{C})_{y}}}_{\left(\sigma_{y}\right)}\end{array}$ & $\begin{array}{c}\overline{(\mathrm{O}-\mathrm{C})_{\alpha}} \\
\left(\sigma_{\alpha}\right) \\
\end{array}$ & $\begin{array}{c}\overline{(\mathrm{O}-\mathrm{C})_{\delta}} \\
\left(\sigma_{\delta}\right) \\
\end{array}$ & $\bar{N}_{\text {obs }} \quad \bar{N}_{\text {stars }}$ \\
\hline $\begin{array}{l}0 .^{\prime} 006 \\
\left.(0)^{\prime} 047\right)\end{array}$ & $\begin{array}{c}0.007 \\
(0 ., 055)\end{array}$ & $\begin{array}{c}0 . !^{\prime} 043 \\
\left(0 !^{\prime} 030\right)\end{array}$ & $\begin{array}{l}-0 .^{\prime} 012 \\
\left(0 !^{\prime} 019\right)\end{array}$ & 743 \\
\hline
\end{tabular}

(Jacobson 1989). The JPL Development planetary Ephemeris DE405 used in this work are available electronically from the JPL Horizons on-line solar system data and ephemeris computation service (Giorgini et al. 1996).

With the star positions (measured and from the corrected catalog) in the field of Uranus observations, a least-squares procedure based on a Householder transform (Lawson \& Hanson 1974), was used to determine the parameters of scale and orientation. Were used these parameters to determine the observed positions of the satellites of Mars, in the 1995 observations. In Table 3, the astrometric calibration results are shown for Ariel, Umbriel, Titania, and Miranda, the four major satellites of Uranus (Veiga et al. 1999). The mean and standard deviation of the observed minus calculated positions, $X=\Delta \alpha \cos \delta$ and $Y=\Delta \delta$, referred to Oberon are shown.

Was applied the same procedure for the 2003 observations of Pluto. Those results show, in Table 3, that the parameters, scale and orientation, were determinated with good accuracy. Unfortunately, several Mars observations in the years 1998, 1999, and 2001 cannot be reduced because no other planetary observations with stars in the field for astrometric calibration were available.

Starting from the parameters determined in the stellar fields of Uranus and Pluto, the standard deviation of all residuals, observed minus calculated positions, for the two satellites of Mars are: for 141 positions of Phobos was obtained $\sigma_{\bar{\alpha}}=00^{\prime} 058$ and $\sigma_{\bar{\delta}}=0$.'053. For 185 positions of Deimos, was obtained $\sigma_{\bar{\alpha}}=$

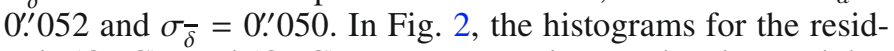
uals $(\mathrm{O}-\mathrm{C})_{\alpha}$ and $(\mathrm{O}-\mathrm{C})_{\delta}$, are presented. It can be observed that the residuals for the satellites have distributions similar to a normal distribution. The standard deviation of a Gaussian fit to the Phobos residuals is equal to $\sigma_{\alpha, \delta}=00^{\prime} 072$. The same for Deimos residuals is $\sigma_{\alpha, \delta}=0$ ' $^{\prime} 060$.
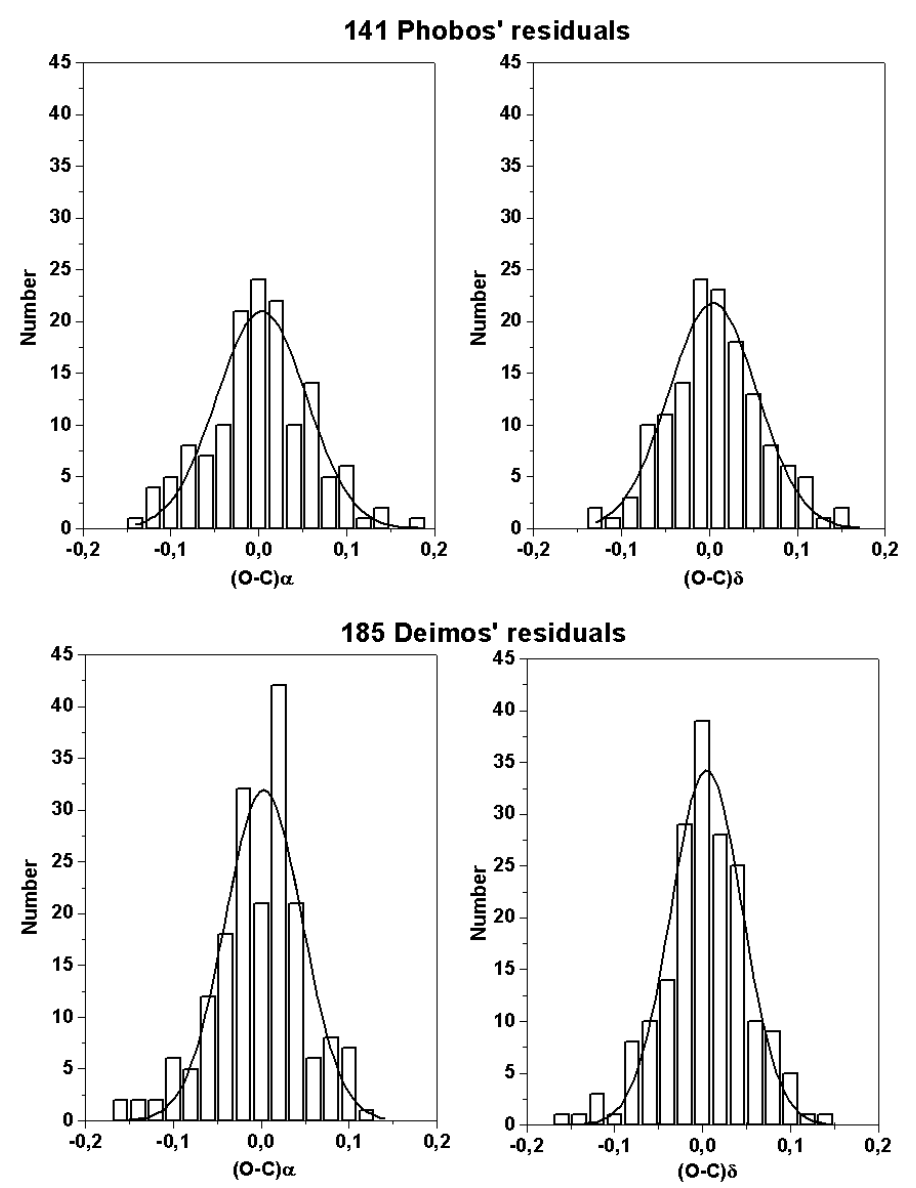

Fig. 2. Histogram for the right ascension and declinations residuals, in arcseconds, of Martian satellites. The $(\mathrm{O}-\mathrm{C}) \alpha$ and $(\mathrm{O}-\mathrm{C}) \delta$ are $\left(\alpha_{\text {observed }}-\alpha_{\text {calculated }}\right)$ and $\left(\delta_{\text {observed }}-\delta_{\text {calculated }}\right)$, respectively.

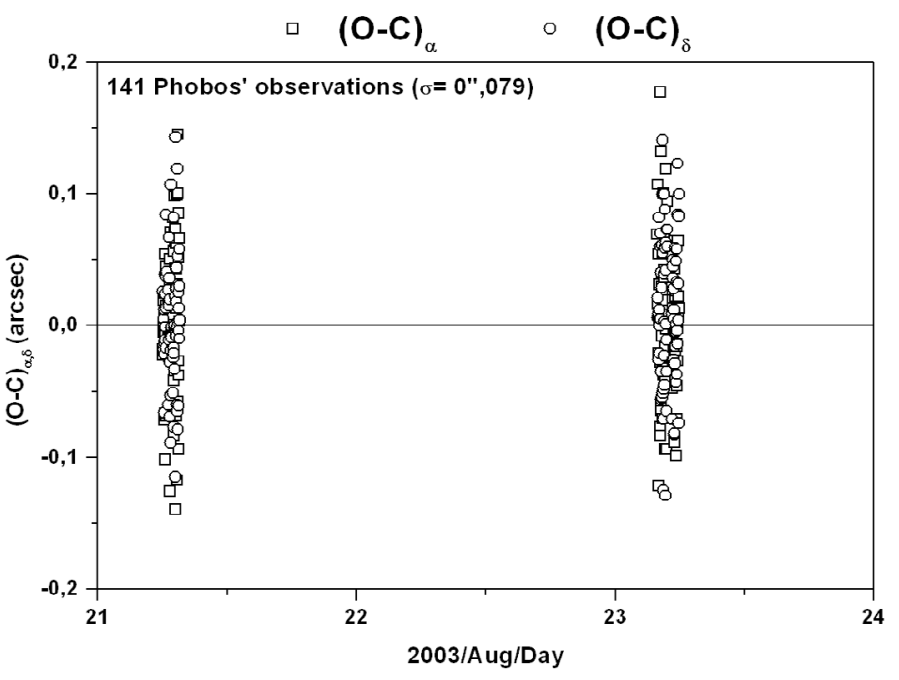

Fig. 3. Residuals $(\mathrm{O}-\mathrm{C})_{\alpha, \delta}$, in arcseconds, for the Phobos satellite as a function of time.

\section{Final comments}

In Figs. 3 and 4, the $(\mathrm{O}-\mathrm{C})$ distribution of residuals are presented with respect to the date of the observation. Comparing the positions obtained here with the orbit calculated by Jacobson, the $\mathrm{O}-\mathrm{C}$ residuals are similar to the best observations of Phobos and Deimos from Earth-based observations. This result shows that the CCD observations and the measure and astrometric 

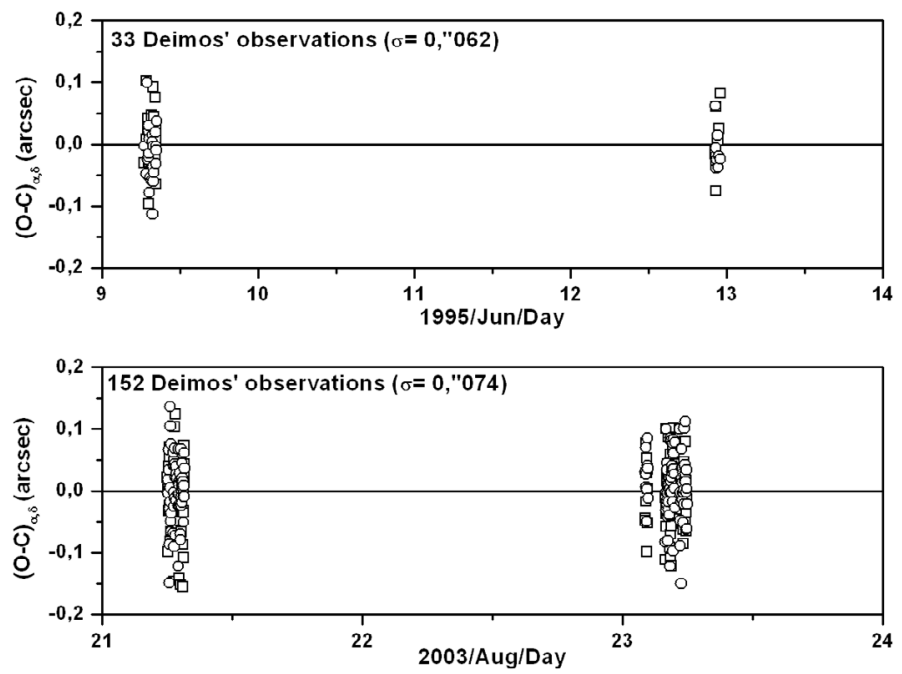

Fig. 4. Residuals $(\mathrm{O}-\mathrm{C})_{\alpha, \delta}$, in arcseconds, for the Deimos satellite as a function of time.

calibration system together allowed us to determine precise positions. We can see, in Figs. 3 and 4, that in the distribution of residuals no bias is verified, showing a good agreement with the JPL ephemeris. The maximum amplitude of residuals is smaller than 0.2 . The results for the observations made in 1995 and
2003 present residuals as good as those presented in the good results published (Sinclair 1972; Morley 1989). The result of these observations can contribute to improved accuracy of the ephemerides and can help in the study of the origin of those satellites.

Acknowledgements. The author thanks all the colleagues at the Laboratorio Nacional de Astrofísica/MCT for their efforts and help during these difficult observations.

\section{References}

Bevington, P. R. 1969, Data Reduction and Error Analysis for The Physical Sciences (New York, USA: MacGraw-Hill Inc.)

Bourget, P., Veiga, C. H., \& Vieira Martins, R. 2001, ASP, 113, 436

Chapman, C. R., Veverka, J., Thomas, P. C., et al. 1995, Nature, 374, 783

Colas, F. 1992, A\&AS, 96, 485

Chiu, L. T. G. 1977, Astrometric Techniques with a PDS microdensitometer

Giorgini, J. D., Yeomans, D. K., Chamberlin, A. B., et al. 1996, BAAS, 28, 25.04 Jacobson, R. A., Synnott, S. P., \& Campbell, J. K. 1989, A\&A, 225, 548

Lawson, C. L., \& Hanson, R. J. 1974, Solving Least Squares Problems (Englewood Cliffs: Prentice-Hall)

Lynch, D. K., Russell, R. W., Rudy, R. J., et al. 2007, AJ, 134, 1459

Lyot, B. 1939, MNRAS, 99, 580

Morley, T. A. 1989, A\&AS, 77, 209

Oberst, J., Matz, K. D., Roatsch, T., et al. 2006, A\&A, 447, 1145

Sinclair, A. T. 1972, MNRAS, 155, 249

Veiga, C. H. 2006, A\&A, 453, 349

Veiga, C. H., \& Vieira Martins, R. 1995, A\&AS, 111, 387

Veiga, C. H., \& Vieira Martins, R. 1999, A\&AS, 138, 247

Veillet, C., \& Ratier, G. 1980, A\&A, 86, 342 